\title{
Alterstice
}

Revue internationale de la recherche interculturelle

International Journal of Intercultural Research

Revista International de la Investigacion Intercultural

\section{Entre idéologie et réalité, entre bilinguisme parfait et éducation plurilingue : le paradoxe de la Vallée d'Aoste}

\section{Isabelle Puozzo Capron}

Volume 2, numéro 1, 2012

Stratégies de (re)médiation en situation plurilingue. Études de cas en contextes de recherche et d'éducation

URI : https://id.erudit.org/iderudit/1077555ar

DOI : https://doi.org/10.7202/1077555ar

Aller au sommaire du numéro

Éditeur(s)

Alterstice

ISSN

1923-919X (numérique)

Découvrir la revue

Citer cet article

Puozzo Capron, I. (2012). Entre idéologie et réalité, entre bilinguisme parfait et éducation plurilingue : le paradoxe de la Vallée d’Aoste. Alterstice, 2(1), 79-88. https://doi.org/10.7202/1077555ar
Résumé de l'article

La Vallée d'Aoste est une région bi-/plurilingue de l'Italie qui revendique un bilinguisme franco-italien grâce à son histoire. Cependant, dans le système éducatif, le poids historico-culturel du passé engendre une recherche du bilinguisme comme " addition " parfaite de deux langues alors que la littérature scientifique a démontré toute la richesse de la compétence plurilingue " déséquilibrée ". À partir de deux enquêtes, il s'agit d'observer les conséquences d'un ancrage bilingue traditionnel sur le système éducatif et sur des apprenants quant à eux plurilingues. 


\section{7}

alterstice

Revue Internationale

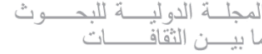

Revista International

de la Investigacion Intercultural

Revista Internacional

de Pesquisa Intercultural

International Journal

de la Recherche Interculturelle

ARTICLE THÉMATIQUE

Entre idéologie et réalité, entre bilinguisme parfait et éducation plurilingue : le paradoxe de la Vallée d'Aoste

Isabelle Puozzo Capron ${ }^{1}$

\section{Résumé}

La Vallée d'Aoste est une région bi-/plurilingue de l'Italie qui revendique un bilinguisme franco-italien grâce à son histoire. Cependant, dans le système éducatif, le poids historico-culturel du passé engendre une recherche du bilinguisme comme " addition " parfaite de deux langues alors que la littérature scientifique a démontré toute la richesse de la compétence plurilingue "déséquilibrée ». À partir de deux enquêtes, il s'agit d'observer les conséquences d'un ancrage bilingue traditionnel sur le système éducatif et sur des apprenants quant à eux plurilingues.

\section{Rattachement des auteurs}

${ }^{1}$ Haute École Pédagogique, Lausanne, Suisse

\section{Correspondance}

isabelle.puozzo@hepl.ch

\section{Mots clés}

perfection; valorisation; plurilinguisme déséquilibré; compétence; sentiment d'efficacité personnelle; insécurité linguistique

\section{Pour citer cet article :}

Puozzo Capron, I. (2012). Entre idéologie et réalité, entre bilinguisme parfait et éducation plurilingue : le paradoxe de la Vallée d'Aoste. Alterstice, 2(1), 79-88. 


\section{Introduction}

De nombreux territoires revendiquent actuellement une éducation bi-/plurilingue, s'inscrivant théoriquement dans la philosophie du Cadre Européen Commun de Référence pour les Langues (CECR) (Conseil de l'Europe, 2001). Pourtant, une observation approfondie montre souvent une pratique cloisonnée de la didactique et un ancrage dans une aspiration au mythe du bilinguisme comme addition parfaite de deux langues (Causa, 2009), c'est-à-dire la maîtrise équilibrée de deux langues. Alors que la littérature scientifique prône depuis de nombreuses années la valorisation de la compétence plurilingue déséquilibrée, c'est-à-dire un profil différent de compétences d'une langue à l'autre, il semblerait que les enseignants refusent encore de voir le déséquilibre comme une richesse et non un handicap à l'apprentissage. Le cas de la Vallée d'Aoste (VDA) n'échappe pas à l'exception. Les problèmes convergeant autour de la question du bilinguisme vont cependant au-delà de la question du système éducatif, mais touchent avant tout à son Statut spécial acquis sur des fondements historiques. Ce territoire est actuellement partagé entre l'idéologie d'un bilinguisme parfait et la réalité, à savoir que ce bilinguisme parfait n'existe pas et que tous les acteurs y gagneraient à concevoir une compétence plurilingue déséquilibrée dans une éducation plurilingue.

Les grandes lignes historiques permettront de mieux comprendre et expliquer le bilinguisme valdôtain. Le système éducatif sera aussi décrit pour montrer comment se renforce l'idée d'une addition parfaite entre l'italien et le français, notamment à l'épreuve certificative de français du secondaire en VDA, soit la 4 é épreuve. Pour comprendre les conséquences d'un manque de valorisation et d'application de la compétence plurilingue déséquilibrée, deux perspectives seront présentées. La première est celle d'une enquête quantitative sur les langues menée par Puolato (2006); le titre de cet article reprend partiellement celui de son ouvrage. La seconde est l'enquête que j'ai réalisée, dans une démarche empirico-inductive, sur le sentiment d'efficacité personnelle [SEP] linguistique à l'entrée (classe de $1^{\mathrm{e} 1}$ ) et à la sortie du lycée (classe de $5^{\mathrm{e}}$ ). L'objectif est de faire émerger des résultats les problèmes convergeant autour de la $4^{\mathrm{e}}$ épreuve, celle de français, dite " 4 épreuve de français", unique sur le territoire italien.

\section{La Vallée d'Aoste, une région bi-/plurilingue}

\section{Le contexte}

La VDA est une région autonome du nord de l'Italie. Son Statut spécial a été obtenu grâce à son histoire et son particularisme linguistique, à savoir un bilinguisme franco-italien. Si aujourd'hui plus personne ne parle le français dans les rues d'Aoste, avant l'unification de l'Italie en 1861, les Valdôtains utilisaient effectivement le français et le dialecte franco-provençal, appelé patois, comme langues de communication. À partir de la formation de l'État italien, une revendication de la valdostanéité s'est affirmée par la défense de la langue française (Omezzoli, 1995) contre l'introduction progressive de l'italien et son officialisation. Durant l'époque fasciste, la disparition du français a connu son apogée (1919-1945) (Omezzoli, 1965/1966) puisque « la politique linguistique visait l'éradication de toute différence linguistique sur le territoire national » (Cavalli, 2005, p. 51). Le "traumatisme linguistique » vécu par le peuple valdôtain est donc une véritable "séparation de la mère ", la langue française, qui a abouti à un " déracinement » linguistique et identitaire (Benrabah, 1999, p. 65). La résistance menait donc un combat physique et linguistique. Il faut attendre la fin de la guerre et la publication de la Constitution italienne en 1948 pour que son Statut spécial lui soit conféré, reconnaissant ainsi sa particularité linguistique avec un enseignement paritaire des deux langues et une utilisation du français dans l'administration publique. Toutefois, le bilinguisme actuel pose encore de nombreuses interrogations. La description du système plurilingue de la VDA oscille entre différentes perspectives. Le regard interne à la situation n'est pas toujours des plus objectifs; le regard extérieur offre en revanche une description plus proche des faits. Par exemple, Tabouret-Keller (2006) décrit synthétiquement l'insertion du bilinguisme institutionnel. Une première impression de perfection s'en dégage; elle conclut justement son article sur les résultats de l'enquête de la Fondation Émile Chanoux sur la langue maternelle: "Italian is

\footnotetext{
${ }^{1}$ Dans le système éducatif italien, les élèves entrent au lycée à l'âge de 14 ans et le cycle des études est quinquennal. À la sortie, en $5^{\mathrm{e}}$ année, les élèves ont donc 19 ans.
} 
mentioned by only 63 percent [...], Franco-Provençal [...] 35 percent, and French by only 1 percent $^{2}$ » (p. 675). La précédente description parfaite s'écroule alors avec ces dernières lignes. II est donc intéressant d'avoir une lecture ethnographique hétérogène afin de comprendre les différentes subtilités de la situation valdôtaine.

\section{Un bilinguisme institutionnel}

La VDA présente donc un bilinguisme institutionnel puisque le français est utilisé dans le système éducatif et dans l'Administration publique. Pour chaque concours, il faut au préalable passer un examen de langue française qui évalue les quatre compétences (production-compréhension/écrite-orale). L'employé administratif perçoit sur son salaire une indemnité mensuelle dite de "bilinguisme" pour l'utilisation plus ou moins fréquente du français durant l'exercice de ses fonctions.

Le système éducatif délivre aussi une éducation bilingue dans le sens où " due (o più) lingue vengono usate come veicolo per l'insegnamento delle varie materie scolastiche " (Balboni, 1999, p. 34). À l'école, le français est pratiqué de la maternelle au lycée. Les programmes officiels régionaux prévoient une découverte ludique de la langue, et ce dès la petite enfance. À partir de l'école primaire, le programme de français s'articule progressivement sur l'apprentissage de l'écrit. De plus, toutes les matières doivent prévoir des séquences d'apprentissage dans cette langue. Pourtant, $20 \%$ des enseignants déclarent « di non parlare mai in francese, neppure a scuola ${ }^{4}$ "(Puolato, 2006, p.18). Cette déclaration semble paradoxale puisque tous les employés perçoivent au même titre une indemnité de bilinguisme qui implique un devoir envers la communauté. Au collège et au lycée, le nombre d'heures de français est identique à celui d'italien et des budgets sont mis à la disposition des établissements scolaires pour la réalisation de projets bilingues. Les politiques linguistiques éducatives s'insèrent dans un projet plurilingue tout au long de la scolarité. Depuis 1998, avec la réforme sur l'Esame di Stato (examen de fin d'études secondaires) en Italie, les épreuves de français ont été repensées en VDA et la 4 é épreuve (quarta prova) de français a été instaurée afin de proposer une épreuve à l'Esame di Stato incluse dans ce continuum et qui exonère ensuite les élèves de l'épreuve de langue française de tous les concours de l'administration publique régionale.

\section{La $4^{e}$ épreuve de français}

Un calque superficiel masquant des divergences d'épreuves

L'épreuve de français est la copie conforme de l'épreuve d'italien qui est, quant à elle, nationale. Les apprenants peuvent choisir entre trois types d'épreuve : I'analyse littéraire, la rédaction-dissertation, l'analyse-production (une étude comparative entre deux textes et une iconographie).

L'analyse littéraire en français correspond à l'analisi del testo en italien et repose sur une structure identique, avec des questions de compréhension, d'analyse textuelle et une production. La rédaction-dissertation correspond au tema avec un premier sujet littéraire en français et historique en italien et un second sur un thème d'actualité dans les deux langues. Ce mot composé rédaction-dissertation est quelque peu confus. La pratique des professeurs de français est de présenter l'épreuve de la dissertation sans s'interroger sur l'ambiguïté du mot composé. En effet, la rédaction désigne généralement une épreuve plutôt créative, pratiquée au collège, sans structure canonique précise. Ce mot composé renvoie alors à des représentations opposées: tâche collégienne/tâche lycéenne, absence de règles/règles canoniques, sujet d'imagination/sujet dialectique, etc. La méthodologie d'enseignement en est d'autant plus obscure (Puozzo Capron, 2011). Enfin, l'analyse-production correspond au saggio breve. Toutefois, pour le saggio breve, les élèves peuvent choisir entre la rédaction de ce type d'épreuve ou d'un article de journal, alors qu'en français la deuxième possibilité est exclue. Or sans cette deuxième opportunité, l'épreuve est réduite à une dimension uniquement scolaire. En choisissant de rédiger un article de journal, l'élève a l'occasion de mettre en œuvre la dimension communicative de l'écriture. L'article de journal ne requiert pas de structuration textuelle académique alors qu'une analyse comparative repose toujours sur une dialectique traditionnelle. Enfin,

\footnotetext{
${ }^{2}$ " l'italien est choisi à $63 \%$ uniquement [...], le franco-provençal [...] à $35 \%$, et le français à seulement $1 \%$ »

3 " deux langues (ou plus) sont utilisées comme langue véhiculaire de l'enseignement des différentes matières scolaires ».

4 " de ne jamais parler en français, même pas à l'école ".
} 
les fondements épistémologiques du saggio breve n'apparaissent pas en français. En effet, le saggio breve s'inscrit dans une lignée éditoriale, comme l'article, mais il est de type argumentatif (Mola, 2000, p. 170). Alors que l'article de journal informe et peut relever de la chronique, le saggio breve doit « descrivere, indicare, esporre e spiegare ». On entre directement au cœur du problème, dans le vif du sujet sans passer par une introduction, comme l'exige la dissertation. Ainsi, le calque ne demeure ici qu'une illusion due à une transposition certes identique des documents, mais avec une consigne radicalement différente.

\section{Problèmes convergeant autour de la $4^{e}$ épreuve de français}

La $4^{\mathrm{e}}$ épreuve de français en VDA relève d'un niveau taxinomique très élevé. L'épreuve de français se revendique comme la soi-disant copie conforme de celle d'italien (Conseil de l'Europe, 2009), mais ce parallélisme soulève de nombreux doutes. En effet, l'apprentissage de la littéracie est certes similaire entre les deux langues, mais les compétences demeurent déséquilibrées puisque la pratique communicative du français se limite aux murs de l'école, voire au professeur de français uniquement. Cette limite du bilinguisme institutionnel (Baker, 2008) fait que la langue est donc décontextualisée de sa fonction première (Cummins, 2006). Par ailleurs, le Profil régional de la politique linguistique éducative. Région autonome Vallée d'Aoste (Conseil de l’Europe, 2009) précise que le niveau de français est supérieur à celui d'une langue étrangère, mais que les compétences entre les deux langues sont asymétriques. De plus, la langue maternelle « entretient [...] un certain nombre de qualités intrinsèques, en particulier celles d'être maîtrisée avec le plus haut degré de compétence et de permettre d’exprimer sa pensée de la façon la plus précise possible »(Castellotti, 2007, p. 21-22). L'identité bilingue se trouve alors en situation de crise dans la mesure où il est demandé au jeune adulte, à l'issue du système scolaire, de savoir exprimer une pensée complexe à l'écrit avec un bagage linguistique certes d'un bon niveau, mais demeurant néanmoins inférieur à celui de la langue maternelle. La frustration se fait alors ressentir puisque le jeune bilingue n'arrive pas toujours à formuler sa pensée dans la langue cible.

Des compétences inégales n'impliquent pas forcément une attitude négative face à l'apprentissage du français. Or le Rapport régional (Decime et Vernetto, 2007) cite une étude qui montre que $27 \%$ des jeunes générations voudraient se passer du français. Pourquoi un tel refus? Pourquoi une vision si négative de la langue et du bilinguisme asymétrique? Comment se fait-il que la richesse plurilingue ne soit pas perçue? Les objectifs fixés ne sont-ils pas trop élevés sachant que les compétences sont asymétriques? Quelle image la dimension du calque véhicule-t-elle auprès de l'ensemble des acteurs sociaux de l'école? Le paradoxe même de ce système éducatif est de tenter de construire une éducation plurilingue tout en niant la spécificité de cette compétence plurilingue au moment de l'évaluation de l'épreuve de français à l'Esame di Stato.

En outre, il résonne, notamment derrière les mots "dissertation " et " analyse littéraire ", une idée d'enseignement/apprentissage maternel qui serait peut-être à remettre en discussion dans l'apprentissage d'une langue étrangère ou seconde. De plus, ces types d'épreuves découlent essentiellement de la tradition académique française. Ces représentations culturelles vont influencer à la fois la méthode d'enseignement de la production comme structure canonique et les critères d'évaluation, qui se basent sur la norme de la culture scolaire française et non sur l'approche actionnelle du CECR, alors que les élèves français sont scolarisés dans un système monolingue et que les élèves valdôtains bilingues vivent, eux, dans deux «cognitive worlds » différents (Bialystok, 2001, p. 88) et que leurs expériences linguistiques ne sont donc pas identiques, et ne peuvent mener aux mêmes performances.

\section{Deux enquêtes pour creuser le paradoxe}

Pour dépasser l'aspect descriptif, deux enquêtes sur les langues en Vallée d'Aoste seront à présent décrites, celle de Puolato (2006) et la mienne (Puozzo Capron, 2009 b). 
Entre idéologie et réalité. L'enquête de Puolato (2006)

Le titre de cet ouvrage marque d'emblée le ton : Francese-italiano, italiano-patois: il bilinguismo in Valle d'Aosta fra realtà e ideologia. La réalité linguistique y est décrite en se basant à la fois sur des documents historiques, scientifiques, ethnographiques etc., et sur une recherche menée par la chercheure qui dévoile progressivement l'idéologie de cette région. Seules les données sur le couple franco-italien sont décrites et non pas celles sur le dialecte ou l'anglais. Le choix d'un tel titre souligne, au premier abord, une certaine ambiguïté. En effet, la première dénotation du substantif idéologie dans Le Dictionnaire historique de la langue française (Rey, 2000) est la suivante : " une science nouvelle ayant pour objet l'étude des idées, au sens général de faits de conscience, de leur origine, de leurs caractères et de leurs lois »(p.1062). Dans la mesure où l'auteur cherche à mesurer les compétences dans les différentes langues, cette première définition semble à exclure. La seconde définition, apparue avant 1800, est dans la continuité de la première, «idéologie appartient à l’histoire de la philosophie ». Le travail de Puolato relève plutôt de l'ethnographie et non de la philosophie. Vers 1800, « un sens péjoratif et qu'il a conservé " apparaît : " discussions, analyses portant sur des abstractions sans rapport avec les faits et la réalité positive » (Rey, 2000, p. 1062). Puolato reprend sans doute cette connotation du terme " idéologie " puisqu'elle l'oppose à celui de réalité. Son étude vise justement à monter que les discours officiels sur la situation linguistique sont très éloignés de la réalité "sans rapport avec les faits ». Une dernière définition du mot idéologie est à préciser: "ensemble d’idées qui inspire un gouvernement, un parti » (Rey, 2000, p. 1062). Au moment des élections, tous les partis valdôtains prévoient une petite partie de leur programme, rédigée en français, où ils défendent des valeurs conservatrices et identitaires de la langue. Ce point ne fait bien sûr pas l'objet d'étude de Puolato puisque sa recherche a été menée dans des écoles auprès d'élèves et d'enseignants. Toutefois, on note combien en Vallée d’Aoste les discours politiques et linguistiques ont partie liée.

Par ailleurs, le contexte communicatif où l'on utilise le français, précise Puolato (2006), demeure l'école, même si cette langue a perdu " la sua funzione di lingua simbolo del particolarismo e della cultura valdostana ${ }^{5}$ " (p. 38$)$. Alors qu'en 1965/1966, Omezzoli désignait la langue française comme "un oggetto mitico ${ }^{6}$ " (p. 17), représentation d'un idéal autonomiste et qui connote l'Antiquité, la beauté et la poésie, Puolato arrive quant à elle en 2006, donc quarante années plus tard, à la conclusion finale que le français n'est pas une langue fantôme, mais une langue " "congelata" e, in quanto tale, sempre uguale a se stessa " " (p. 348-349). Cette métaphore est particulièrement forte pour désigner la situation valdôtaine. Si celle du fantôme donne l'idée d'une entité bien présente, qui hante et qui fait peur; celle de la congélation souligne, en revanche, la rigidité, la stérilisation, l'atemporalité. Cela semble quelque peu excessif puisque le français est bien présent dans les salles de classe et dans les bureaux de l'Administration. Les stéréotypes qui lui sont effectivement liés sont chargés " d'idee inculcate, di ideologie trasmesse aprioristicamente » (p. 350) et dans ce sens uniquement la métaphore de la congélation peut prendre son sens. Le français est donc directement lié au patrimoine historique, linguistique, à la notion d'autonomie et de patrie valdôtaine (Omezzoli, 1965/1966), alors que la notion de valdostanéïté semble aujourd'hui majoritairement abandonnée au profit de l'italianité, notamment chez les jeunes de 18 à 30 ans (Puolato, 2001).

De plus, deux grandes questions sont soulevées à un moment donné de sa recherche :

« fino a che punto si può sostenere (o credere) che una lingua veicoli significativi culturali e identitari, se la comunità non avverte il bisogno di servirsene spontaneamente, per esprimere e affermare, con l'uso stesso della lingua, quei valori ad essa affidati? In base alla situazione linguistica attuale, quale posto spetta alla Valle d'Aosta nel composito mondo francofono? " (Puolato, 2006, p. 49).

\footnotetext{
5 " sa fonction de langue symbolique du particularisme et de la culture valdôtains »

${ }^{6}$ " un objet mythique »

7 " "congelée" et, en tant que telle, toujours égale à elle-même"

${ }^{8}$ "Jusqu'à quel point peut-on soutenir (ou croire) qu'une langue véhicule des signifiants culturels et identitaires, si la communauté ne ressent pas le besoin de s'en servir spontanément, pour exprimer et affirmer, avec l'usage même de la langue, les valeurs attribuée à celle-ci Sur la base de la situation linguistique actuelle, quelle place revient à la Vallée d’Aoste dans le monde francophone composite?"
} 
Dans sa première question, Puolato parle de "ressentir le besoin » de s'exprimer en français pour exprimer son identité. Quelle est la valeur d'une langue que personne n'utilise dans son quotidien? Le français est souvent imposé; les élèves sont censés développer leurs compétences orales en s'exprimant uniquement dans cette langue, même si cela n'est pas une règle obligatoire et varie d'un professeur à l'autre. Si on laisse le choix aux apprenants, certains s'expriment spontanément en français, d'autres demandent la permission d'exprimer leurs idées en italien, mais la plupart choisissent l'italien. Ses premiers résultats font ressentir d'emblée une incohérence puisque « [...] entrambe le lingue godono di parità giuridica e sono lingue di prestigio ${ }^{9}$ " et pourtant on relève " la prevalenza pressoché assoluta dell'italiano ${ }^{10}$ " (Puolato, 2006, p. 129-130). L'adjectif "absolu » renforce véritablement l'impression d'une négation de la compétence partielle en français, même de la part d'apprenants d'origine valdôtaine. Puolato s'interroge également sur la question de l'alternance codique. En effet, selon elle, l'essence même du discours bilingue est de pouvoir passer d'un code à l'autre alors que « [...] i risultati ottenuti non rilevano alternanza di codice $[\ldots]^{11} »($ p. 241-242). La frustration demeure " [...] nell'incapacità del français di soddisfare in pieno le funzioni potenzialmente connesse al suo status di lingua ufficiale ${ }^{12}$ " (p. 277). Elle ne remet certes pas en cause le statut du français, mais elle souligne le fait que les Valdôtains, même s'ils ne considèrent pas le français comme une langue maternelle, ne la reconnaissent pas non plus comme " una lingua seconda » (p.179), alors que selon elle, ce statut de langue seconde pourrait être une solution pour penser l'éducation plurilingue. Ce changement de perspective du français permettrait à l'enseignement bilingue d'être moins chargé d'attentes inadéquates pour les enseignants et les apprenants qui ne ressentiraient plus ce poids d'une perfection jamais atteinte. Les enseignants ne devraient ainsi plus chercher à préparer tous les élèves (du lycée général au professionnel) à un examen final demandant les compétences nécessaires pour mener à bien une dissertation et une analyse littéraire comme si le candidat était un locuteur natif français. Les objectifs et méthodes pourraient ainsi être revus et s'inscrire plus fortement dans la philosophie du CECR (Conseil de l'Europe, 2001). Les apprenants n'en seraient pas moins bilingues et ils se percevraient certainement plus riches et épanouis.

\section{Une compétence plurilingue avec une forte composante francophone mais qui reste dévalorisée}

L'apprentissage des langues a connu un nouveau tournant avec la publication du CECR (Conseil de l'Europe, 2001), notamment pour la compétence plurilingue qui est valorisée même lorsqu'elle aboutit à des formes d'interférences dans les activités langagières de production. On n'attend plus la performance parfaite, mais l'interférence est signe d'un apprentissage en devenir. Actuellement, l'apprentissage de l'oral passe par la méthode communicative et l'approche par compétences (Beacco, 2007). Durant la performance orale, les enseignants acceptent l'interlangue ou l'alternance codique dans le discours. De nombreux progrès sont néanmoins à faire au niveau de la compétence écrite, puisque ces interférences deviennent pénalisantes dans un contexte d'évaluation. L'apprentissage de l'écrit se fait encore sous une norme académique française avec le poids de la sanction, le refus de l'interlangue ou même de l'alternance codique. Ce fossé entre l'oral et l'écrit met l'apprenant en situation d'incompréhension et de dévalorisation de la compétence bilingue qui reste ancrée dans l'image de l'addition de deux langues et de la perfection. Avoir choisi en VDA des épreuves identiques entre l'italien et le français renforce cette idée d'un apprentissage maternel de la langue seconde. Les questions de notre recherche sont donc les suivantes:

- vu que le système leur fait passer des épreuves identiques, les apprenants valdôtains ont-ils une perception déséquilibrée ou équilibrée de leurs compétences en français et en italien?

- la VDA présente-t-elle une situation de bilinguisme parfait ou de plurilinguisme déséquilibré?

Ce n'est pas une évaluation en termes de niveau de compétences ou de réussite, mais de perception des compétences. Une évaluation des compétences aurait été inutile dans la mesure où les recherches sur le territoire montrent bien des compétences asymétriques. Néanmoins, l'idéologie linguistique en VDA et l'implicite qui se cache derrière une évaluation symétrique à l'Esame di Stato peuvent laisser croire que les apprenants perçoivent véritablement une symétrie de leurs compétences. Par conséquent, passer des épreuves symétriques entre les deux langues ne poserait aucun souci. Cette enquête mesure donc le sentiment d'efficacité personnelle (SEP)

\footnotetext{
9 " les deux langues jouissent d'un même statut juridique et elles sont langues de prestige »

${ }^{10}$ "la dominance presque absolue de l'Italie »

${ }^{11}$ "les résultats obtenus ne relèvent pas de l'alternance des codes »

12 " dans l'incapacité du français de satisfaire pleinement les fonctions potentielles liées à son statut de langue officielle »
} 
linguistique. Le SEP « refers to beliefs in one's capabilities to organize and execute the courses of action required to produce given attainments ${ }^{13}$ " (Bandura, 1997, p. 3). II désigne les croyances d'un individu en ses compétences à atteindre des buts. II s'agit donc de mesurer la perception que l'apprenant a de ses compétences pour réaliser une tâche sur une échelle de mesure de 0 à 100. L'article ne présente qu'une partie des résultats.

La figure 1 montre qu'en italien, on observe une constance dans la perception des compétences actives et passives orales et de la compétence passive écrite avec des résultats élevés, environ $80 \%$. On a une faible diminution, un écart de 5 points environ lorsqu'il s'agit de la production écrite en $1^{\mathrm{e}}$. Entre les deux langues, on observe une perception inégale de la compétence. On est donc bien dans le cadre d'une compétence plurilingue déséquilibrée au sens positif du terme et non pas dans une situation d'un bilinguisme parfait et équilibré. En français, au niveau de la production orale, une diminution est notable avec un écart de 13 points, qui pourrait s'expliquer par une tendance des programmes à se focaliser sur la compétence écrite en vue de la préparation à la $4^{\mathrm{e}}$ épreuve de français. En revanche, pour ce qui est de la compétence passive, à l'écrit et à l'oral, on constate une augmentation de la perception avec un résultat prouvant un SEP satisfaisant ${ }^{14}$. Cette augmentation montre qu'il est dommage que le système n'évalue pas la compréhension écrite et orale par deux épreuves distinctes selon le modèle du

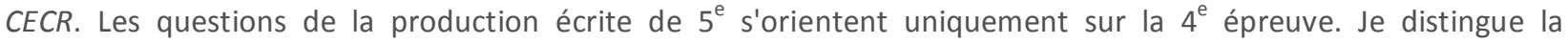
compétence d'analyse littéraire qui correspond à un type d'épreuve, la compétence dialectique qui renvoie à la dissertation et la compétence argumentative qui appartient à la fois à la dissertation et à l'analyse-production. On note en italien que, par rapport au résultat de la $1^{\mathrm{e}}$, la compétence dialectique et celle d'analyse littéraire se maintiennent sur un SEP très satisfaisant. Seul un résultat chute d'une tranche, celui de la compétence argumentative. En français, une seule compétence, la dialectique, se maintient dans la même tranche que celle de la $1^{\mathrm{e}}$. Deux compétences, celle d'analyse littéraire et celle argumentative, diminuent d'une catégorie, désignant alors un SEP moyen.

Figure 1 : Les compétences en langues en $1^{\mathrm{e}}$ et en $5^{\mathrm{e}}$

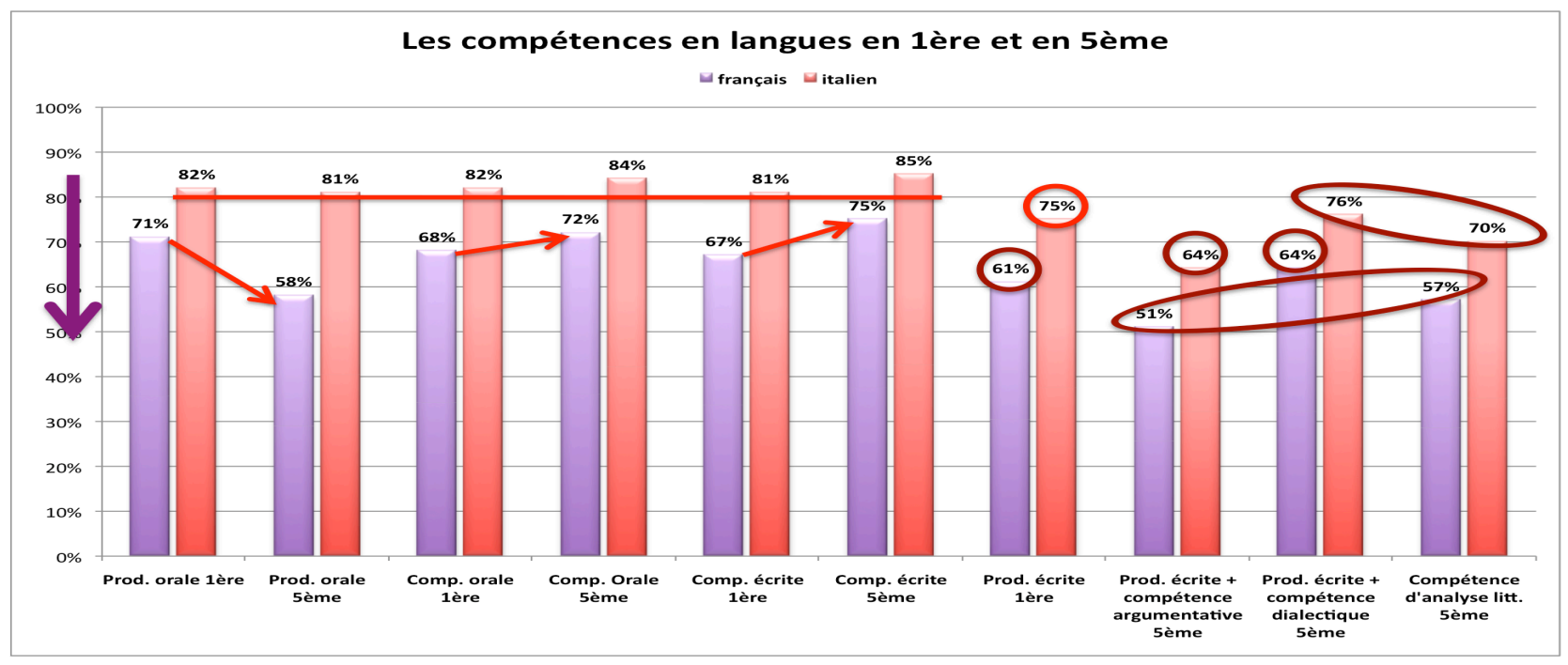

Pour ne pas m'arrêter à cette lecture globale, j'ai voulu creuser ces résultats en analysant la variable nominale de la "filière de l'établissement scolaire ". En Italie, chaque école propose une offre de formation correspondant à un unique cursus. J'ai sélectionné deux lycées: I'Istituto Professionale Regionale Alberghiero (IPRA) (une école hôtelière) pour la formation professionnelle et le lycée classique bilingue pour la formation générale. J'ai donc pris les extrêmes puisque les élèves passent les mêmes épreuves de français, mais dans des cursus de prestige très

\footnotetext{
13 «L'efficacité personnelle perçue concerne la croyance de l'individu en sa capacité d'organiser et d'exécuter la ligne de conduite requise pour produire des résultats souhaités » (Bandura, 1997, p. 12).

${ }^{14}$ L'évaluation du SEP s'effectue sur une taxonomie de huit tranches (voir Puozzo Capron, 2009b).
} 
différents. La section bilingue du lycée classique est inspirée du lycée européen (Puozzo Capron, 2009a). Toutes les matières y sont enseignées en français. En revanche, le lycée hôtelier délivre une formation professionnelle qui se décompose en deux cycles. Durant les trois premières années, les élèves doivent préparer un examen professionnel intermédiaire qui leur permet de choisir d'entrer directement dans le monde du travail ou bien de poursuivre pendant deux années et de passer le baccalauréat. En français, cet examen consiste en une épreuve écrite et/ou orale qui varie d'un lycée professionnel à l'autre. Donc les trois premières années sont censées aboutir à la préparation uniquement de cette épreuve et de la langue de spécialité. Officiellement, les professeurs de français ne doivent pas commencer la préparation à la 4 épreuve de français avant la $4{ }^{\mathrm{e}}$ année. Officieusement, il est nécessaire de commencer avant, de manière détournée les deux premières années et la $3^{\mathrm{e}}$ année de manière explicite. Ces deux établissements proposent donc des dispositifs basés l'un sur la compétence littéraire et l'autre sur une compétence professionnelle. Alors pourquoi ces apprenants se retrouvent-ils à passer les mêmes épreuves de français en fin de parcours? Leurs compétences linguistiques sont-elles véritablement valorisées de manière paritaire dans ces épreuves?

La figure 2 renvoie à la production écrite à l'entrée du lycée où il y a déjà un écart au départ avec un SEP moyennement faible en français (48\%). En $5^{\mathrm{e}}$, on constate un écart allant de 9 à 34 points, soit environ $42 \%$ d'écart entre les deux formations (voir figure 3). La donnée que je tenais simplement à mettre en évidence est le $40 \%$ pour la compétence argumentative. On est donc bien loin du $51 \%$ de notre histogramme précédent. Ces apprenants ont déjà une perception différente à l'entrée au lycée. Cependant, ces cinq années de lycée qui sont censées aboutir au même examen, donc à la même formation (voire formation accentuée en professionnelle pour rattraper le niveau), montrent que la préparation à cette épreuve ad hoc de l'Esame di Stato ne fait que renforcer la différence au lieu de la diminuer.

Figure 2 : La compétence active écrite en $1^{\mathrm{e}}$

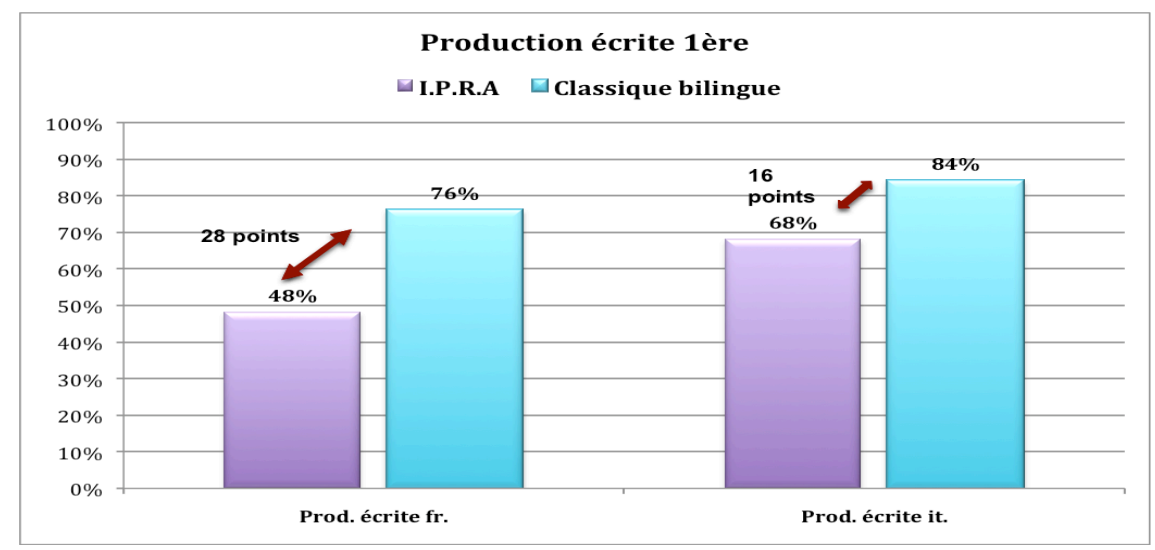

Figure 3 : La compétence active écrite en $5^{\mathrm{e}}$

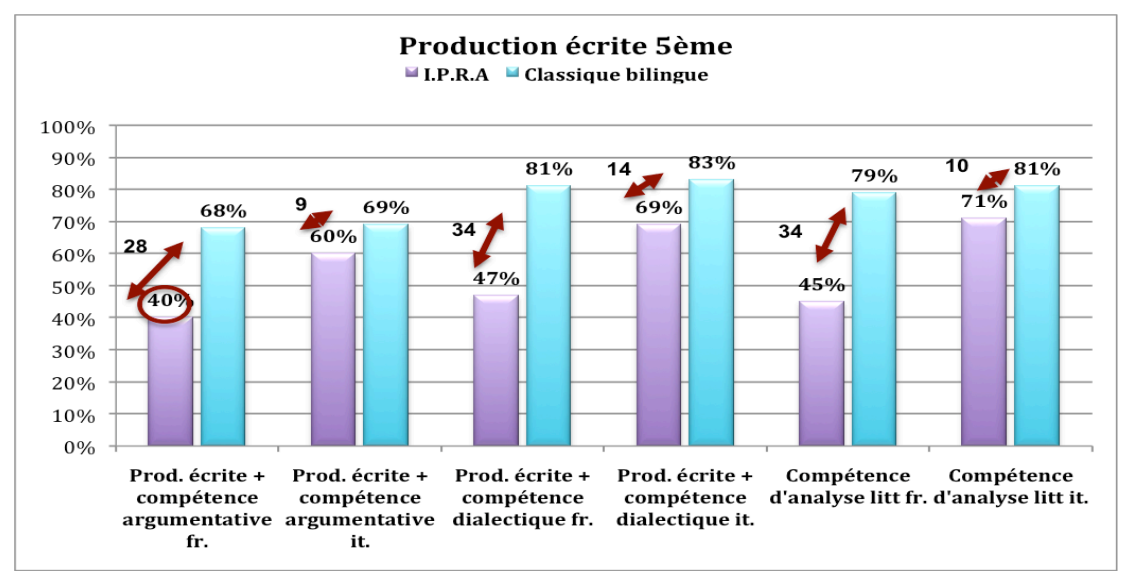




\section{Conclusion}

L'enquête de Puolato et la mienne montrent que l'idéologie consistant à croire en une compétence bilingue parfaite et équilibrée engendre une évaluation calquée sur cette dernière. Plus on avance dans le système scolaire, plus les objectifs sont élevés et moins les apprenants se perçoivent compétents (en tout cas pour la production écrite). L'ombre du bilinguisme parfait obscurcit l'horizon des apprenants et les empêche de s'épanouir dans un contexte d'éducation bi-/plurilingue européen. L'analyse plus approfondie de mes données montre également une situation véritablement préoccupante pour la formation professionnelle. Ce sont des élèves qui entrent au lycée avec un niveau inférieur et à qui l'on demande de rattraper en cinq années leur différence, leurs lacunes et de se préparer au même niveau que la formation générale sans véritablement leur en donner la possibilité puisque la préparation à cette épreuve est condensée en deux années officiellement - officieusement en trois années au lieu de cinq. Ces élèves, souvent en difficulté dans des matières générales, doivent produire le double d'effort par rapport à ceux de la formation générale. Cette inégalité des chances va à l'encontre même de l'éthique de tout système éducatif européen actuel. Une école hôtelière vise d'abord la préparation à la langue de spécialité. Toujours dans un esprit de valorisation des compétences, "même des apprenants dits faibles peuvent être valorisés dans leurs compétences tout à fait présentes, même si elles sont moins élevées que celles d'autres apprenants " (Piccardo, Berchoud, Cignatta, Mentz et Pamula, 2009, p. 2). Les élèves d'un lycée hôtelier risquent d'avoir une préparation littéracique inférieure à ceux d'un lycée classique bilingue. II ne s'agit pas ici de les considérer comme des apprenants déficitaires, mais de prendre conscience que les compétences qu'ils développent durant leur parcours formatif quinquennal sont de l'ordre d'une compétence linguistique professionnelle et qui ne sont en rien valorisées dans une épreuve telle que la dissertation ou l'analyse littéraire.

De manière générale, des épreuves comme celles qui ont été choisies en VDA véhiculent encore plus l'imaginaire du bilingue parfait. La compétence plurilingue asymétrique est étouffée derrière cette aspiration à la perfection. La question idéologique actuelle fait perdre de vue la réalité valdôtaine et, à mon avis, il ne s'agit pas uniquement de la place de la VDA dans le monde francophone, comme s'interrogeait Puolato, mais plutôt au niveau européen. Dans la situation valdôtaine, la réflexion ne se situe pas uniquement au niveau éducatif, mais avant tout sur le plan législatif et quant au statut du français. Une nécessaire évolution de la définition des objectifs plurilingues ne doit pas être perçue comme un abandon du bilinguisme, ce qui pourrait entraîner d'importantes conséquences sur l'économie, la politique, etc. La question politique ne doit pourtant pas nous rendre aveugles sur les problèmes qui convergent dans le système éducatif, il est temps de penser prioritairement à la formation des élèves, pour qu'ils puissent tirer parti de leur scolarisation dans une région frontalière qui préfigure l'Europe plurilingue de demain.

\section{Références bibliographiques}

Baker, C. (2008). Foundations of Bilingualism Education and Bilingualism ( $4^{\mathrm{e}}$ éd.). New York: Multilingual matters.

Balboni, P. (1999). Dizionario di glottodidattica. Perugia : Guerra.

Bandura, A. (1997). Self-efficacy : The exercise of control. New York : Freeman and Compagny.

Beacco, J.-C. (2007). L'approche par compétences dans l'enseignement des langues. Paris : Didier.

Benrabah, M. (1999). Langue et pouvoir en Algérie. Histoire d'un traumatisme linguistique. Paris : Séguier.

Bialystok, E. (2001). Bilingualism in development. Language, literacy \& cognition. Cambridge : Cambridge University Press.

Castellotti, V. (2007). La langue maternelle en classe étrangère. Paris : Clé international.

Causa, M. (2009). Enseignement bilingue francophone et apports du CECR. Le français dans le monde, 366, 31-33.

Cavalli, M. (2005). Éducation bilingue et plurilinguisme. Le cas du Val d’Aoste. Paris : Didier.

Conseil de l'Europe. (2001). Cadre Européen Commun de Référence pour les langues : apprendre, enseigner, évaluer. Paris : Didier. 
Conseil de l'Europe. (2009). Profil régional de la politique linguistique éducative. Région autonome Vallée d'Aoste. Italie. Division des Politiques linguistiques Strasbourg. [http://www.coe.int/t/dg4/linguistic/Profils__FRANÇAISasp - TopOfPage].

Cummins, J. (2006). Interdependence of first- and second-language proficiency in bilingual children. Dans E. Bialystok (dir.), Language Processing in Bilingual Children (p. 70-89). Cambridge : Cambridge University Press.

Decime R. et Vernetto, G. (dir.). (2007). Rapport Régional du Profil de la politique linguistique éducative. Vallée d'Aoste. Aosta : Assessorat de l'Education et de la Culture.

Mola, L. (2000). Il nuovo esame du maturità. Milano : Alpha Test.

Omezzoli, T. (1965/1966). Conflitti di lingua e di cultura in Valle d'Aosta (thèse de doctorat, Università degli Studi di Torino).

Omezzoli, T. (1995). Lingue e identità valdostana. Dans Storia d'Italia. Le regioni dall'unità a oggi. La Valle d'Aosta (139-202). Torino : Einaudi.

Piccardo, E., Berchoud, M., Cignatta, T., Mentz, O. et Pamula, M. (2009). ECEP Project Guide. Council of Europe. [Page Web]. Accès : [http://ecep.ecml.at/Resources/tabid/1029/language/en-GB/Default.aspx]

Puolato, D. (2001). Aosta spazio varietetico e sistema di valori identitari : configurazioni a confronto. Dans Fondation Emile Chanoux, Une Vallée d'Aoste bilingue dans une Europe plurilingue (p. 79-87). Aosta : Tipografia Vadostana.

Puolato, D. (2006). Francese-italiano, italiano-patois : il bilinguismo in Valle d'Aosta fra realtà e ideologia. Berne : Peter Lang.

Puozzo Capron, I. (2009a). L'atelier culturel. Le français dans le monde, 366, 36-37.

Puozzo Capron, I. (2009b). L'erreur, un outil d'apprentissage en contexte plurilingue. Le cas de la Vallée d'Aoste. Synergies Algérie, 6, 79-86. [http://ressources-cla.univ-fcomte.fr/gerflint/Algerie6/capron.pdf]

Puozzo Capron, I. (2011). Repenser la 4 épreuve de français en Vallée d'Aoste pour valoriser la compétence bi/plurilingue. Education et sociétés plurilingues, 30, 27-40.

Rey, A. (dir.). (2000). Dictionnaire historique de la langue française. Paris : Le Robert.

Strubell, M. (2001). Implications du sondage pour la planification linguistique dans la Vallée d'Aoste. Dans Fondation Emile Chanoux (dir.), Une Vallée d'Aoste bilingue dans une Europe plurilingue (p. 105-112). Aosta : Tipografia Vadostana.

Tabouret-Keller, A. (2006). Bilingualism in Europe. Dans T. K. Bhatia et W.C. Ritchie (dir.), The handbook of bilingualism (p. 662-688). Oxford : Blackwell Publishing. 